\title{
WEINBAUM'S CONJECTURE ON UNIQUE SUBWORDS OF NONPERIODIC WORDS
}

\author{
ANDREW J. DUNCAN AND JAMES HOWIE
}

(Communicated by Ron Solomon)

\begin{abstract}
Following a conjecture of Weinbaum, we show that every nonperiodic word $W$ of length at least 2 in a free group has a cyclic permutation of the form $U V$, where each of $U$ and $V$ occur precisely once as a cyclic subword of $W$ and neither occurs as a cyclic subword of $W^{-1}$. In fact, we prove a somewhat stronger version of this result and also give a number of applications to one-relator products of groups.
\end{abstract}

\section{INTRODUCTION}

Let $W$ be a cyclically reduced word of length greater than 1 in the free group $F=F(X)$ on some set $X$ and assume that $W$ is not a proper power. In a recent paper [9], Weinbaum showed that some cyclic permutation of $W$ has the form $U V$, where $U$ and $V$ are nonempty cyclic subwords of $W$, each of which occurs exactly once as a cyclic subword of $W$. Indeed, for any letter $x \in X \cup X^{-1}$ that occurs in $W$, he found such a decomposition of $W$ with $U$ both beginning and ending with $x$ and $V$ neither beginning nor ending with $x$. Weinbaum conjectured in [9] that $W$ has a cyclic permutation of the form $U V$ such that $U$ and $V$ are nonempty cyclic subwords of $W$, each occurring exactly once as a cyclic subword and neither $U$ nor $V$ occurring as a cyclic subword of $W^{-1}$.

In this note we show that Weinbaum's conjecture is true and give some applications to the theory of one-relator products of groups. Although Weinbaum's conjecture makes no mention of $U$ beginning and ending with specific letters, we are able to prove a stronger version of the conjecture, which does just that, and so is more in the spirit of the theorem of [9].

Definition. Let $W$ be a cyclically reduced word in the free group $F$ on a basis $X$. A (cyclic) subword $U$ of $W$ is said to be uniquely positioned if (i) no other cyclic subword of $W$ is identically equal to $U$ and (ii) no cyclic subword of $W^{-1}$ is identically equal to $U$.

Theorem. Let $W$ be a cyclically reduced word in $F$ of length greater than 1 that is not a proper power, and let $x \in X$ be a letter such that either $x$ or $x^{-1}$

Received by the editors January 29, 1991.

1991 Mathematics Subject Classification. Primary 20F05, 20F06, $20 \mathrm{~F} 10$.

Research supported by SERC grant GR/E 88998. 
(or both) occurs in $W$. Then one of the following holds:

(i) $W$ has a cyclic permutation of the form $x^{s} P x^{t} P^{\varepsilon}$, where $P$ is a nonempty word containing neither $x$ nor $x^{-1}, s, t$ are nonzero integers with $s= \pm t$, and $\varepsilon= \pm 1 ;$ or

(ii) $W$ has a cyclic permutation of the form $U V$, where $U, V$ are nonempty, uniquely positioned subwords, $U$ begins with $x^{ \pm 1}$ and ends with $x^{ \pm 1}$, and $V$ neither begins nor ends with $x^{ \pm 1}$.

Note that if (i) holds then (ii) does not hold. Thus Weinbaum's Theorem in [9] does not generalize in full. However, in (i) the subwords $x^{s} P$ and $x^{t} P^{\varepsilon}$ are uniquely positioned, so in both cases Weinbaum's conjecture is true.

The proof of Weinbaum's conjecture in its original form is more straightforward than that of the above theorem. We give this in $\S 2$. It is similar to Pride's proof [7] that a one-relator group presentation in which the relator is an $n$th power satisfies the small cancellation condition $\mathrm{C}(2 n)$.

In $\S 3$ we apply this result to one-relator products of groups. Suppose $G$ is the quotient of a free product $(A * B)$ of groups by the normal closure of a single word $R=S^{m}$, where $S$ is cyclically reduced of length at least 2 . If $m \geq 4$ then a number of results were proved for $G$ in [4-6], such as the Freiheitssatz: each of $A, B$ embeds in $G$ via the natural homomorphism; Weinbaum's theorem: no nonempty, proper cyclic subword of $R$ represents the identity element of $G$; the Identity Theorem (with some exceptions): the normal closure of $R$ in $A * B$, made abelian, is isomorphic as a $\mathbf{Z} G$-module to $\mathbf{Z} G /(1-S) \mathbf{Z} G$; and the word problem: if $A$ and $B$ have soluble word problem then so does $G$. A consequence of our result is that all these theorems extend to the case $m=3$, under the additional hypothesis that no element of $A$ or $B$ of order 2 occurs as a letter of $S$.

Finally, in $\S 4$ we prove the above stated theorem in full.

\section{Proof of Weinbaum's conjecture}

Theorem 2.1. Let $W$ be a cyclically reduced word of length greater than 1 in the free group $F(X)$. Then some cyclic permutation of $W$ has the form $U V$ where $U$ and $V$ are nonempty, uniquely positioned cyclic subwords of $W$.

Proof. Let $G$ denote the torsion-free one-relator group $\langle X \mid W\rangle$. Then $G$ is locally indicable [1, 3], so right-orderable [2]. Choose a right ordering $<$ on $G$. Suppose $W=y_{1} \cdots y_{n}$, where $y_{i} \in X \cup X^{-1}$. For $1 \leq i \leq n$, let $g_{i}$ denote the element of $G$ represented by the initial segment $y_{1} \cdots y_{i}$ of $W$. Then it follows from a result of Weinbaum [8] that the $g_{i}$ are pairwise distinct elements of $G$. Let $\mu=\min \left\{g_{1}, \ldots, g_{n}\right\}$ and $M=\max \left\{g_{1}, \ldots, g_{n}\right\}$ (with respect to the chosen right ordering of $G$ ), and let $S_{\mu}, S_{M}$ denote the corresponding initial segments of $W$. Then $W$ has a cyclic permutation of the form $U V$, where either $S_{M}=S_{\mu} U$ or $S_{\mu}=S_{M} V$ (depending on which of $S_{\mu}, S_{M}$ is longer). We claim that $U$ and $V$ are uniquely positioned. To see this, suppose that $U$ is identical to the subword of $W$ beginning with the $(i+1)$ st letter and ending with the $j$ th. Then, computing in $G$, we have $g_{i}^{-1} g_{j}=\mu^{-1} M$. Since $g_{i} \geq \mu$, we have

$$
g_{j}=g_{i}\left(g_{i}^{-1} g_{j}\right) \geq \mu\left(g_{i}^{-1} g_{j}\right)=\mu\left(\mu^{-1} M\right)=M,
$$

with equality only if $g_{i}=\mu$. Hence $g_{i}=\mu$ and $g_{j}=M$. Similarly $V$ is identical to no other cyclic subword of $W$. If $U$ (say) is identical to a cyclic 
subword $C$ of $W^{-1}$ then a similar argument shows that $C$ must be $V^{-1}$. But $U$ is not identical to $V^{-1}$ since $W$ is reduced. Hence $U$ and $V$ are uniquely positioned, as claimed.

\section{Applications}

Let $A, B$ be groups, and $R=S^{m} \in A * B$, where $S$ is cyclically reduced of length at least 2, and not a proper power, and $m$ is a positive integer. Assume also that no letter occurring in $S$ has order 2 in $A$ or $B$. Let $Y$ denote the (finite) subset of $A \cup B$ consisting of those letters that occur in $S$ or in $S^{-1}$. Then $Y=Y^{-1}$ and no element of $Y$ is its own inverse. Hence there is a subset $X$ of $Y$ with the property that $X \cup X^{-1}=Y$ and $X \cap X^{-1}=\varnothing$. If we reinterpret $S$ as a word in $F(X)$ and apply Theorem 2.1, we see that, up to cyclic permutation, $S$ has the form $U V$, where $U$ and $V$ are nonempty, uniquely positioned subwords of $S$.

If $m \geq 4$ then a number of results were proved in [4-6] about the onerelator product group $G=(A * B) / N(R)$, where $N$ denotes normal closure. Under the additional assumption that $S$ contains no letter of order 2, we show how to improve that to $m \geq 3$. We require a lemma about pictures over $G$, which is essentially just Pride's observation that the word $R$ satisfies the small cancellation condition $\mathrm{C}(2 \mathrm{~m})$ [7]. We first recall some concepts concerning pictures over a one-relator product. For further details we refer the reader to [4-6].

A picture $\Gamma$ over $G$ (on $D^{2}$ ) consists of a finite collection of disjoint small disks $v_{1}, \ldots, v_{n}$ (called vertices) in int $D^{2}$, together with a properly embedded 1-submanifold of $D^{2} \backslash$ int $\{$ vertices (whose components are called the arcs of $\Gamma$ ) and a labelling function that assigns to each corner (that is, each component of $\partial v_{i} \backslash$ \{edges $\}$ or of $\partial D^{2} \backslash\{$ edges $\}$ ) a label (an element of $A \cup B$ ).

The vertex label of $v_{i}$ is the product of the labels at $v_{i}$, read anticlockwise. This is required to be $R^{ \pm 1}$ in cyclically reduced form (up to cyclic permutation). A region of $\Gamma$ is a component of $D^{2} \backslash \Gamma$. If we assume that $\Gamma$ is connected and meets $\partial D^{2}$ then all regions are simply connected. The label of a region $\Delta$ is the product of the corner labels of $\Delta$, read clockwise, and is required to be $1_{A}$ or $1_{B}$.

Two arcs that together bound a region are said to be parallel. In particular, the two corner labels are mutually inverse. We also use the term parallel for the equivalence relation generated by this relation. Thus a class of $k$ parallel arcs identifies two subwords of $R^{ \pm 1}$. A pair of vertices of $\Gamma$ cancel if they are joined by an arc $e$ and their vertex labels read from the end points of $e$ are mutually inverse. A picture with no cancelling pair of vertices is reduced.

Lemma 3.1. If $v$ is a vertex in a reduced picture on $D^{2}$ over $G$ that is not connected to $\partial D^{2}$ by arcs then there are at least $2 m$ classes of parallel arcs incident at $v$.

Proof. We may assume that $S=U V$ as above. Then of the arcs incident at $v$ there are $2 m$ special ones, dividing the vertex label $R$ up into alternate $U$ and $V$ segments. Suppose that two of these belong to the same parallel class of $\operatorname{arcs} \beta$, say. Then $\beta$ identifies some subword $Q$ of $R$, containing $U$ or $V$, with a subword $Q^{\prime}$ of $R$ or $R^{-1}$ (see [4]). Since $U$ and $V$ are uniquely 
positioned subwords of $S$, it follows that $Q$ is also uniquely positioned. Hence $\beta$ represents a proper cancellation between $v$ and an oppositely oriented vertex $u$ of the picture (in particular $u \neq v$ ). This contradicts the assumption that the picture was reduced.

Recall from [4-6] that we associate to any picture $\Gamma$ on $D^{2}$ a tessellation of $S^{2}$ by shrinking each vertex to a point, removing all arcs going to $\partial D^{2}$, shrinking each remaining class of parallel arcs to a single edge, and then embedding $D^{2}$ in $S^{2}$. To each corner of this tessellation we associate an angle of $(k-2) \pi / k$ (where the corner belongs to a $k$-sided region). We use these notions in the proofs of the following corollaries.

Corollary 3.2 (Isoperimetric inequality). If $m \geq 3$ and some reduced (connected) picture on $D^{2}$ over $G$ has $j$ interior vertices and $b$ boundary vertices, then $j<b^{2} / \pi^{2}$.

Proof. The tessellated 2-sphere $T$ induced from this picture has the property that each interior vertex of $\Gamma$ has nonpositive combinatorial curvature in $T$ (in the sense that the incident angles add up to at least $2 \pi$; this follows since there are at least six incident corners, and the minimum corner angle is $\pi / 3$ ). The result follows by [6, Corollary 2.4].

Corollary 3.3. If $m \geq 3$ and $\Gamma$ is a reduced picture on $D^{2}$ over $G$ such that at most three vertices of $\Gamma$ are connected to $\partial D^{2}$ by arcs, then all vertices of $\Gamma$ are connected to $\partial D^{2}$ by arcs.

This is a special case of Conjecture $\mathrm{F}(3)$ of [4].

Proof. As in the proof of Theorem F(5) in [4] it is enough to consider the case of a connected picture with no separating vertices such that the corresponding tessellation has no cycles of length 3 or less, other than the boundaries of triangular regions. The Euler Formula tells us that the sum, over all vertices, of the incident combinatorial angles is $(2 v-4) \pi$, where $v$ is the total number of vertices. If there are $b$ boundary vertices then the sums of the incident angles is at most $2(b-2) \pi$ (since each interior vertex has at least six neighbours, by Lemma 3.1, and hence angle sum at least $2 \pi$ ). Since boundary vertices not connected to $\partial D^{2}$ by arcs have at least six neighbours, they have angle sum at least $5 \pi / 3+(b-2) \pi / b$, whereas vertices connected to $\partial D^{2}$ by arcs have angle sum at least $\pi / 3+(b-2) \pi / b$. An easy calculation now shows that the number of boundary vertices connected to $\partial D^{2}$ by arcs exceeds the number not connected to $\partial D^{2}$ by at least 3 .

Hence $b=3$, with each boundary vertex of $\Gamma$ connected to $\partial D^{2}$ by arcs and having angle sum precisely $2 \pi / 3$. In particular, these three boundary vertices have only each other for neighbours, and so $\Gamma$, being connected, has no vertices other than those three.

As explained in [4], the following results are consequences of Corollary 3.3. In all cases, we assume that $G=(A * B) / N\left(S^{m}\right)$, with $m \geq 3$, where $S$ is cyclically reduced of length at least 2 , containing no letters of order 2 .

Theorem 3.4 (The Freiheitssatz). The natural homomorphisms $A \rightarrow G$ and $B \rightarrow G$ are injective.

Theorem 3.5 (Weinbaum's Theorem). No nonempty, proper subword of $S^{m}$ represents the identity element of $G$. 
Corollary 3.6. The word $S$ represents an element of order $m$ in $G$.

Theorem 3.7 (The Identity Theorem).

$$
N\left(S^{m}\right) /\left[N\left(S^{m}\right), N\left(S^{m}\right)\right] \cong \mathbf{Z} G /(1-S) \mathbf{Z} G
$$

as a (right) ZG-module.

Corollary 3.8. There are natural isomorphisms for all $q \geq 3$;

$$
\begin{aligned}
H^{q}(G ;-) & \rightarrow H^{q}(A ;-) \times H^{q}(B ;-) \times H^{q}\left(\mathbf{Z}_{m} ;-\right), \\
H_{q}(G ;-) & \leftarrow H_{q}(A ;-) \oplus H_{q}(B ;-) \oplus H_{q}\left(\mathbf{Z}_{m} ;-\right) ;
\end{aligned}
$$

a natural epimorphism

$$
H^{2}(G ;-) \rightarrow H^{2}(A ;-) \times H^{2}(B ;-) \times H^{2}\left(\mathbf{Z}_{m} ;-\right) ;
$$

and a natural monomorphism

$$
H_{2}(G ;-) \leftarrow H_{2}(A ;-) \oplus H_{2}(B ;-) \oplus H_{2}\left(\mathbf{Z}_{m} ;-\right) .
$$

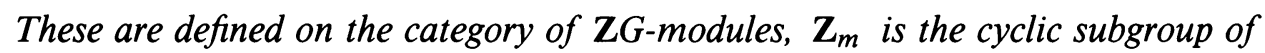
order $m$ generated by $S$, and all these maps are induced by restriction on each factor.

Finally, as in [6] we can use the isoperimetric inequality, Corollary 3.2 , to solve the word problem for $G$ if it is soluble for $A$ and $B$.

Theorem 3.9. If $A$ and $B$ are given by recursive presentations with soluble word problem, then so is $G$. Moreover, the generalized word problem for $A$ (and $B$ ) in $G$ is soluble with respect to these presentations.

The generalized word problem for a subgroup is the algorithmic problem of recognizing whether a given word in the generators represents an element of the subgroup, and if so, of finding a word in the generators of the subgroup that represents the same element.

\section{Proof of Main Theorem}

Theorem. Let $W$ be a cyclically reduced word in $F$ of length greater than 1 that is not a proper power, and let $x \in X$ be a letter such that either $x$ or $x^{-1}$ (or both) occurs in $W$. Then one of the following holds:

(i) $W$ has a cyclic permutation of the form $x^{s} P x^{t} P^{\varepsilon}$, where $P$ is a nonempty word containing neither $x$ nor $x^{-1}, s, t$ are nonzero integers with $s= \pm t$, and $\varepsilon= \pm 1 ;$ or

(ii) $W$ has a cyclic permutation of the form $U V$, where $U, V$ are nonempty, uniquely positioned subwords, $U$ begins with $x^{ \pm 1}$ and ends with $x^{ \pm 1}$, and $V$ neither begins nor ends with $x^{ \pm 1}$.

Proof. The proof is by double induction, first on the number of occurrences of $x$ and $x^{-1}$ in $W$, and second on the length of $W$.

Up to cyclic permutation, we have $W=x^{n_{1}} A_{1} \cdots x^{n_{k}} A_{k}$, where $n_{1}, \ldots, n_{k}$ are nonzero integers and $A_{1}, \ldots, A_{k}$ are nonempty words not involving $x^{ \pm 1}$. Let $n=\max \left\{\left|n_{i}\right| ; 1 \leq i \leq k\right\}$. Introduce a new letter $z \notin X$ and replace each occurrence of $x^{n}$ by $z$ and each occurrence of $x^{-n}$ by $z^{-1}$ to obtain a new word $W^{\prime}$ in $X^{\prime}=X \cup\{z\}$. If $n>1$ then we may apply the inductive hypothesis to $W^{\prime}$ (with respect to the letter $z$ ). If (ii) holds for $W^{\prime}$ then it also holds for 
$W$, since subwords $x^{ \pm n}$ of $W$ occur only in the places corresponding to the letters $z^{ \pm 1}$ of $W^{\prime}$ (by maximality of $n$ ). Suppose then that $W^{\prime}=z^{a} P z^{b} P^{\varepsilon}$, with $a, b, \varepsilon \in\{ \pm 1\}$, where $P$ does not involve $z$. If $P$ does not involve $x$ then $W=x^{\alpha} P x^{\beta} P^{\varepsilon}$, with $\alpha, \beta \in\{ \pm n\}$, so (i) holds for $W$. If $P$ has the form $B x^{\gamma} C$, with $C$ not involving $x$, then $C$ is nonempty, by maximality of $n$. Put $U=x^{\alpha} B x^{\gamma}$ and $V=C x^{\beta} P^{\varepsilon}$. Now $B x^{\gamma}$ cannot be identical to an initial segment of $P^{-1}$, for that would force some terminal segment of $B x^{\gamma}$ to be identical to its own inverse. Hence $U$ can fail to be uniquely positioned in $W$ only if $\alpha=\beta$ and $\varepsilon=1$, which is ruled out by the assumption that $W$ is not a proper power. Similarly, $V$ is uniquely positioned, so (ii) holds for $W$.

Hence we may assume that $n=1$ (so that $n_{i}= \pm 1$ for each $i$ ). Next choose some word $B \in\left\{A_{1}, \ldots, A_{k}\right\}$ of maximal length. Once again, we take a new letter $y \notin X$ and replace $A_{i}$ by $y$ whenever $A_{i}=B$ and by $y^{-1}$ whenever $A_{i}=B^{-1}$ to obtain a new word $W^{\prime \prime}$ in $X \cup\{y\}$. If $B$ has length greater than 1 then $W^{\prime \prime}$ is shorter than $W$. If $A_{j} \neq B^{ \pm 1}$ for some $j$, then there are fewer occurrences of $y^{ \pm 1}$ in $W^{\prime \prime}$ than there are of $x^{ \pm 1}$ in $W$. In either case we may apply the inductive hypothesis to $W^{\prime \prime}$ with respect to the letter $y$. Arguing as above (using the maximality of the length of $B$ ) we see that (ii) holds for $W$ if it holds for $W^{\prime \prime}$. Thus we may assume that $W=C B C^{\alpha} B^{\beta}$ (up to cyclic permutation), where $B$ does not contain $x, C$ begins and ends with $x$ or $x^{-1}$, and no subword of $C$ is identical to $B^{ \pm 1}$. If $C$ has length 1 then (i) holds for $W$. Otherwise write $C$ as $x^{\gamma} D$ and put $U=C^{\alpha} B^{\beta} x^{\gamma}, V=D B$. As in the previous paragraph, we see that $U$ and $V$ are uniquely positioned, and so (ii) holds for $W$.

Hence we are reduced to the case where only two letters $(x, y$, say) of $X$ occur in $W$, and $W$ has the form

$$
x^{\alpha_{1}} y^{\beta_{1}} \cdots x^{\alpha_{k}} y^{\beta_{k}}
$$

for some $k$, where $\alpha_{i}, \beta_{i} \in\{ \pm 1\}$. If $k \leq 2$ then the result is clear (using the assumption that $W$ is not a proper power). Suppose then that $k>2$. By Theorem $2.1 \mathrm{~W}$ has a cyclic permutation $U V$ where $U$ and $V$ are nonempty, uniquely positioned cyclic subwords of $W$. Moreover, $U=\mu^{-1} M$ in the right ordered group $G=\langle X \mid W\rangle$, where $\mu, M$ are, respectively, the least and greatest of the elements $g_{1}, \ldots, g_{2 k}$ of $G$ (with respect to the right ordering) represented by the initial segments of $W$. This follows from the proof of Theorem 2.1.

If $U$ and $V$ have odd lengths, then one begins and ends with $x$-letters and the other with $y$-letters and (ii) holds. Suppose then that $U$ (and hence $V$ ) has even length. Renumbering if necessary, we may suppose that $U=x^{\alpha_{1}} \cdots y^{\beta_{t}}$ with $t \geq k / 2$. Let $T=x^{\alpha_{1}} \cdots x^{\alpha_{t}}$, the initial segment of $U$ of length $2 t-1$. If $T$ is uniquely positioned in $W$, then (ii) holds (since $y^{\beta_{t}} V$ is certainly uniquely positioned), so we may assume that $T$ is not uniquely positioned. Similarly (ii) holds if the terminal segment $Y$ of $V$ of length $2 k-2 t-1$ is uniquely positioned, so we can assume that $Y$ is not uniquely positioned.

If $T$ is identical to a cyclic subword $X$ of $W^{-1}$ then $X$ cannot intersect $T^{-1}$, for if so then the common segment of $X$ and $T^{-1}$ would be identical to its own inverse. Hence the only possibility is that $X$ is the terminal segment of $V^{-1}$ of length $2 t-1$. (In particular, $U$ and $V$ have the same length.) If, also, $Y$ is identical to a subword of $W^{-1}$, then for the same reasons we 
must have $Y^{-1}$ identical to the terminal segment of $U$ of length $k-1$. We have $\alpha_{i}=-\alpha_{k-i+1}=\alpha_{i+1}=-\alpha_{k-i}$ for $1 \leq i \leq t-1$ and, similarly, $\beta_{i}=$ $-\beta_{k-i+1}=\beta_{i-1}=-\beta_{k-i+2}$ for $t+2 \leq i \leq k$. Without loss of generality, we have $U V=(x y)^{t}(y x)^{-t}$, which is cyclically conjugate to the product of two uniquely positioned subwords $x y x^{-1}$ and $(x y)^{1-t} y^{-1}(x y)^{t-1}$.

Hence (possibly interchanging the roles of $Y$ and $T$ if $k=2 t$ ) we may assume that $T$ is identical to a subword $Z$ of $W$. If $Z$ contains the last letter of $V$, then there is an initial segment $E$ of $Z$ (and hence of $U$ ) identical to a terminal segment of $V$. If $g$ is the element of $G$ represented by $E$, then we have $g_{a} g=\mu$ and $\mu g=g_{b}$ for some $a, b \in\{1, \ldots, 2 k\}$. But $\mu<g_{a}$ so $g_{b}=\mu g<g_{a} g=\mu$, a contradiction. Hence $Z$ does not contain the last letter of $V$. In particular, $U$ is an initial segment of a subword $U^{\prime}$ of $U V$, of which $Z$ is a terminal segment. If $U^{\prime}$ has length $2 t+\lambda-1$ then it has period $\lambda$, in the sense that each letter agrees with the letter appearing $\lambda$ places after it.

Next suppose that $Y$ is identical to a subword of $W$. Arguing as above, that subword cannot contain the first letter of $U$, so $V$ is a terminal segment of a subword $V^{\prime}$ of $U V$, of length $2 k-2 t-1+\kappa$ and period $\kappa$ for some $\kappa$. The words $U^{\prime}$ and $V^{\prime}$ intersect in a common segment of length $\lambda+\kappa-2$ and periods $\lambda$ and $\kappa$. Since $\nu=\operatorname{hcf}(\lambda, \kappa) \geq 2$, it follows that this segment (and hence also $U V$ ) has period $\nu$ (see, e.g., [4, Proposition 1]). In particular, the terminal segment of $U$ of length $\nu$ agrees with the initial segment of $V$ of length $\nu$. If $h$ is the element of $G$ represented by this segment, then we have $g_{c} h=M$ and $M h=g_{d}$ for some $c, d \in\{1, \ldots, 2 k\}$. Since $M>g_{c}$ we have $g_{d}=M h>g_{c} h=M$, a contradiction.

Hence we must have $Y$ identical to a subword of $W^{-1}$ which is necessarily a subword of $U^{-1}$, as we remarked previously. If in fact $Y$ is identical to a subword of $T^{-1}$ then, using the periodicity of $U^{\prime}$, we obtain another copy of $Y$ inside $U^{\prime-1}$, displaced from the original by $\lambda$ letters. This process can be repeated until we obtain a subword identical to $Y^{-1}$ lying inside $U^{\prime}$ but not $T$. If this subword intersects $Y$ then we have a contradiction, and if it does not then its last letter must coincide with the last letter of $U$. In the later case the terminal segment $Y^{\prime}$ of $Y$ of length $2 k-2 t-2$ is identical to the inverse of a subword $Y^{\prime \prime}$ of $T$. Repeating the above argument on $Y^{\prime \prime}$ we again obtain a contradiction, unless $\lambda=2$.

Suppose then, finally that $\lambda=2$. Then, without loss of generality, we have $U V=(x y)^{t} x y^{-1}(y x)^{t+1-k}$, with $t<k \leq 2 t$. If $k>t+1$ then the cyclic subwords $x y^{-1} x^{-1}$ and $y^{-1}(y x)^{t+2-k}(x y)^{t}$ are uniquely positioned, while if $k=t+1$ then $t \geq 2$ since $k>2$, so the subwords $x y^{-1} x$ and $y(x y)^{t-1}$ are uniquely positioned.

This completes the proof.

\section{REFERENCES}

1. S. D. Brodskii, Equations over groups and groups with a single defining relator, Siberian Math. J. 25 (1984), 235-251.

2. R. G. Burns and V. W. D. Hale, A note on group rings of certain torsion-free groups, Canad. Math. Bull. 15 (1972), 441-445.

3. J. Howie, On locally indicable groups, Math. Z. 180 (1982), 445-461.

4. __ The quotient of a free product of groups by a single high-powered relator, I. Pictures, Fifth and higher powers, Proc. London Math. Soc. (3) 59 (1989), 507-540. 
5. _ - The quotient of a free product of groups by a single high-powered relator, II. Fourth powers, Proc. London Math. Soc. (3) 61 (1990), 33-62.

6. _ The quotient of a free product of groups by a single high-powered relator, III. The word problem, Proc. London Math. Soc. (3) 62 (1991), 590-606.

7. S. J. Pride, Small cancellation conditions satisfied by one-relator groups, Math. Z. 184 (1983), 283-286.

8. C. M. Weinbaum, On relators and diagrams for groups with a single defining relator, Illinois J. Math. 16 (1972), 308-322.

9. __ Unique subwords in nonperiodic words, Proc. Amer. Math. Soc. 109 (1990), 615-619.

Department of Mathematics, Heriot-Watt University, Riccarton, Edinburgh EH14 4AS, Great Britain

E-mail address: andrew@cara.ma.hw.ac.uk

E-mail address: jim@cara.ma.hw.ac.uk 